\title{
Detection of Subsurface Facilities \\ Including Non-Metallic Pipe
}

\section{FINAL TECHNICAL REPORT}

September 2001 through March 2003

Principal Author - Mr. Herb Duvoisin

$$
26 \text { May } 2003
$$

DOE Cooperative Agreement DE-FC26-01NT41315

\author{
Submitted By: \\ CyTerra Corporation \\ 85 First Avenue \\ Waltham, MA 02451
}




\section{DISCLAIMER:}

This report was prepared as an account of work sponsored by an agency of the United States Government. Neither the United States Government nor any agency thereof, nor any of their employees, makes any warranty, express or implied, or assumes any legal liability or responsibility for the accuracy, completeness, or usefulness of any information, apparatus, product, or process disclosed, or represents that its use would not infringe privately owned rights. Reference herein to any specific commercial product, process, or service by trade name, trademark, manufacturer, or otherwise does not necessarily constitute or imply its endorsement, recommendation, or favoring by the Untied States Government or any agency thereof. The views and opinions of authors expressed herein do not necessarily state or reflect those of the United States Government or any agency thereof. 


\subsection{ABSTRACT}

CyTerra has leveraged our unique, shallow buried plastic target detection technology developed under US Army contracts into deeper buried subsurface facilities and including nonmetallic pipe detection. This Final Report describes a portable, low-cost, real-time, and userfriendly subsurface plastic pipe detector (LULU- Low Cost Utility Location Unit) that relates to the goal of maintaining the integrity and reliability of the nation's natural gas transmission and distribution network by preventing third party damage, by detecting potential infringements. Except for frequency band and antenna size, the LULU unit is almost identical to those developed for the US Army (see Figure 1.0-1). CyTerra designed, fabricated, and tested two frequency stepped GPR systems, spanning the frequencies of importance (200 to $1600 \mathrm{MHz}$ ), one low and one high frequency system. Data collection and testing was done at a variety of locations (selected for soil type variations) on both targets of opportunity and selected buried targets. We developed algorithms and signal processing techniques that provide for the automatic detection of the buried utility lines. The real time output produces a sound as the radar passes over the utility line alerting the operator to the presence of a buried object. Our unique, low noise/high performance RF hardware, combined with our field tested detection algorithms, represents an important advancement toward achieving the DOE potential infringement goal.

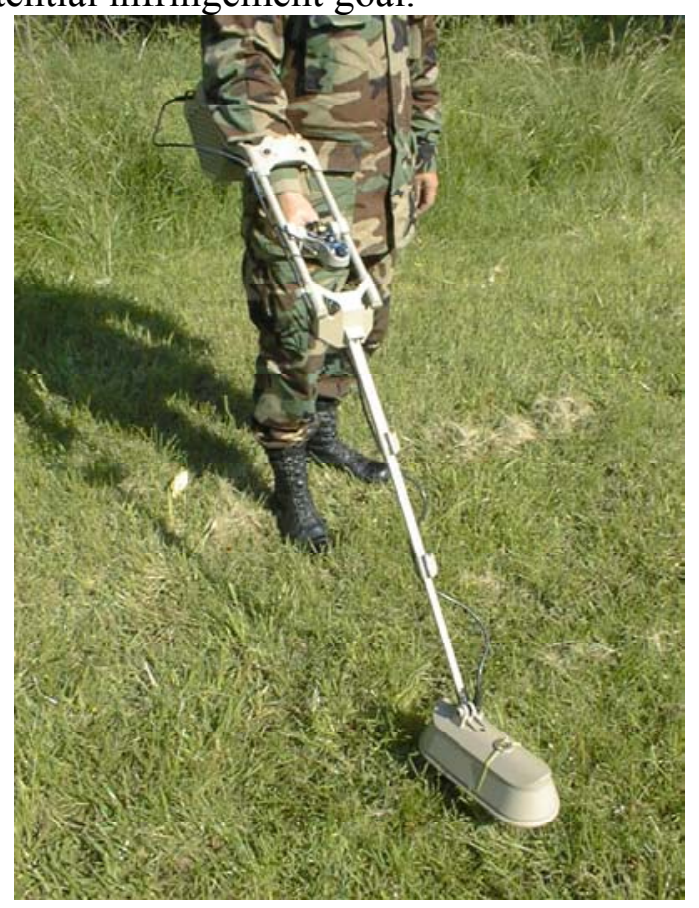

Figure 1.0-1. CyTerra's unique US Army mine performance for the $\mathrm{DOE}$

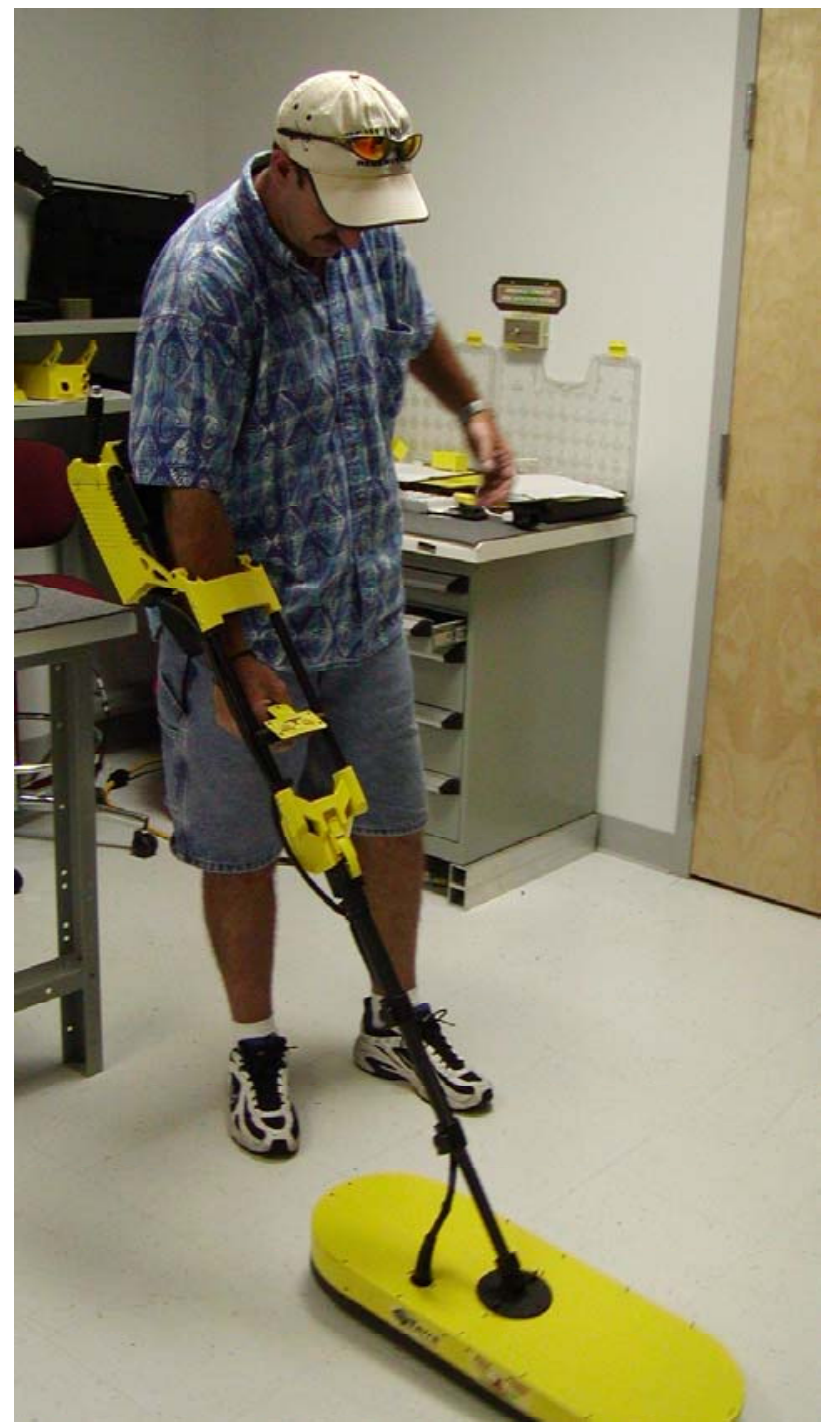

detection experience yielded breakthrough infringement goal. 


\section{Table of Contents}

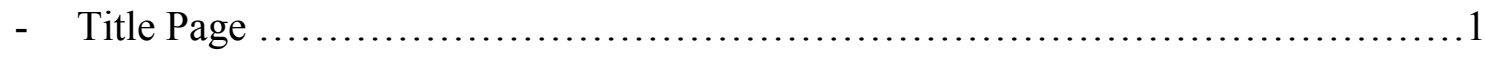

- Disclaimer .............................................................

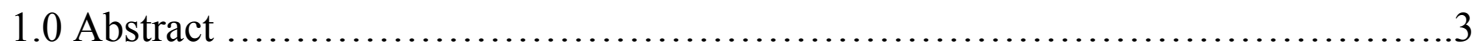

- Table of Contents .........................................................4

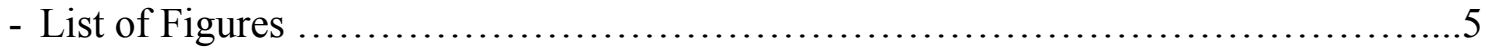

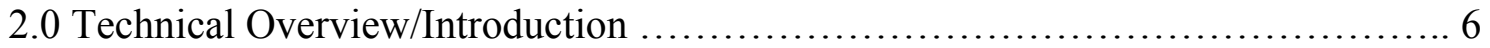

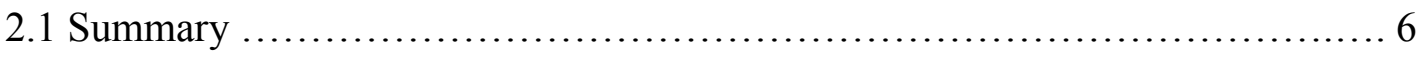

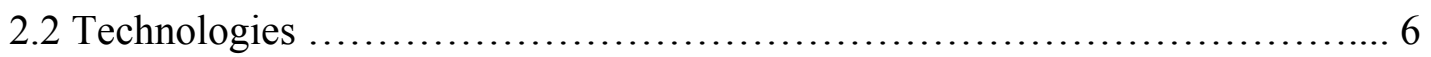

2.3 Breakthroughs .................................................. 8

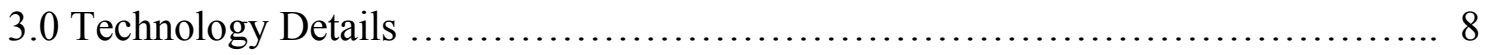

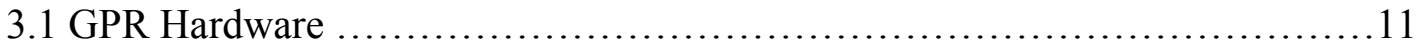

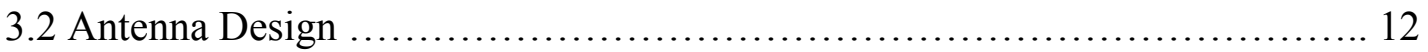

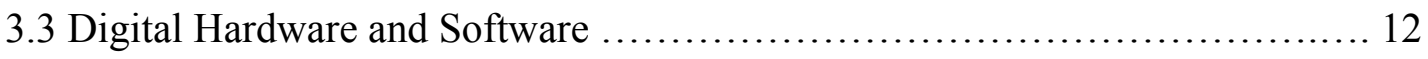

4.0 Tasks and Results .................................................. 13

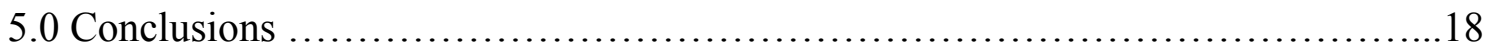




\section{List of Figures}

1.0-1 CyTerra's Unique Experience...........................................4

2.1-1 HSTAMIDS GPR Basis for LULU ....................................6

2.2-1 CyTerra Developed RF Module .........................................

2.2-2 Compact Foldout Detector............................................ 7

3.0-1 Phase I Algorithm demonstrated Accurate Small Diameter Pipe Detection 1-3 ft ..9

3.0-2 Phase I Algorithm Data Processing Results ..................................

3.0-3 LULU Detection 6" Plastic Pipe at 2 foot depth............................. 10

3.1-1 System Electronics Block Diagram................................... 11

3.1-2 LULU System Parameters ............................................12

4.0-1 Sandbox with several Plastic Pipe Targets ................................13

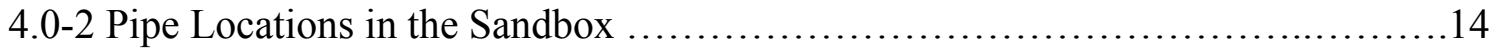

4.0-3 Sandbox Measurements Indicated Good Plastic Pipe Capability ................15

4.0-4 Backyard Trench and Pipes Buried - Post Sandbox Data Collection ..............15

4.0-5 Measurements - Fresh Buried Verses Weathered In Targets....................16

4.0-6 Amplitude Plot Shows Pipe Locations ................................. 16

4.0-7 Backyard Cross-Pol and Co-Pol Depth Plots 4" Plastic Pipe at 4" Depth......... 17

4.0-8 Full Algorithm Audio Alert Show Accurate Pipe Locations ....................17

4.0-9 LULU Operation Over Refilled Trench Shows No False Alarms ................18

5.0-1 Successful User Friendly Buried Pipe Detection Demonstrated .................18 


\subsection{TECHNICAL OVERVIEW}

\subsection{Summary}

The work described relates to the Detection of Subsurface Facilities including Non-Metallic Pipe i.e. a buried pipe detection system developed on Cooperative Agreement DE-FC26$01 N T 41315$ under a cost sharing grant.

CyTerra's portable, low-cost, real-time, and user-friendly subsurface pipe and utility line detector (LULU- Low Cost Utility Location Unit) relates to the goal of maintaining the integrity and reliability of the nation's natural gas transmission and distribution network by preventing third party damage, through the development of sensors to detect potential infringements. Our innovative research and development efforts have resulted in the advanced technologies and improved methodologies to support detection and location of subsurface non-metallic (as well as metallic) pipes. The use of already proven man-portable, user-friendly, plastic mine detection technology (Figure 2.1-1) as a solution for modification to meet DOE goals was key to
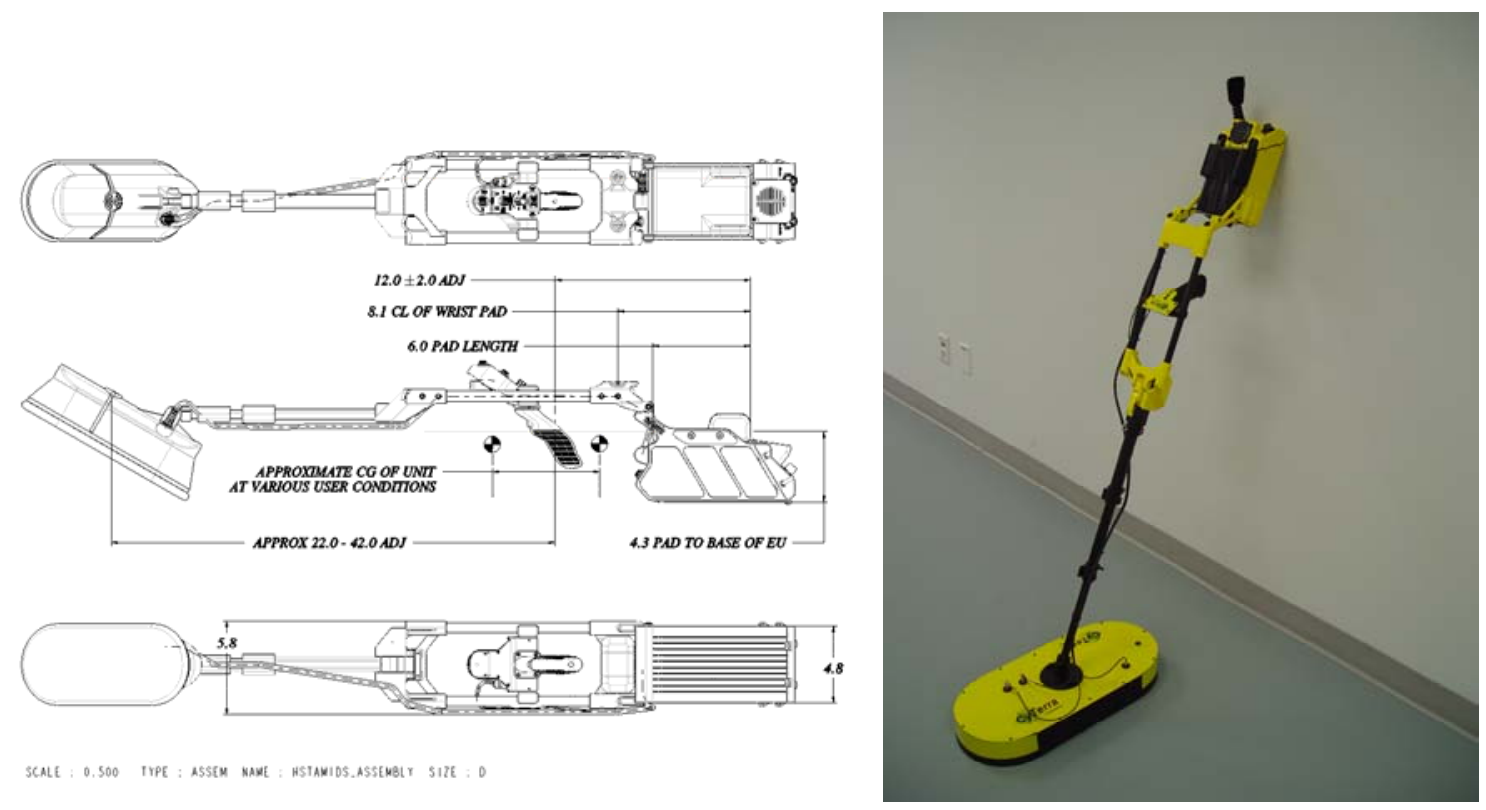

Figure 2.1-1. The $4 \mathrm{~kg}$, handheld GPR unit developed for HSTAMIDS (left) was the basis for the LULU unit (right).

CyTerra's approach. Only minor modifications to the hardware and algorithms produced realtime operator audio feedback when detecting buried plastic or metallic pipes.

\subsection{Technologies}

The work included improvements over existing technologies in the areas of portability, low cost and real-time/user-friendly operation.

Prior technologies for non-metallic pipe detection were expensive, difficult/confusing to use, and provide only after the fact, post-processed information. CyTerra's approach includes: the low-cost hardware implementations developed over the last four years with $\$ 11 \mathrm{M}$ in funding on the HSTAMIDS program (an example is the RF module shown in Figure 1.2-1), the extensive human factors knowledge and development (as shown in Figure 1.2-2), and expedites detection 
by providing real-time feedback (audio feedback) in addition to potential visual postprocessed/mapping feedback (proposed for the follow-on effort).

\section{GPR TRANSCIEVER BLOCK DIAGRAM}

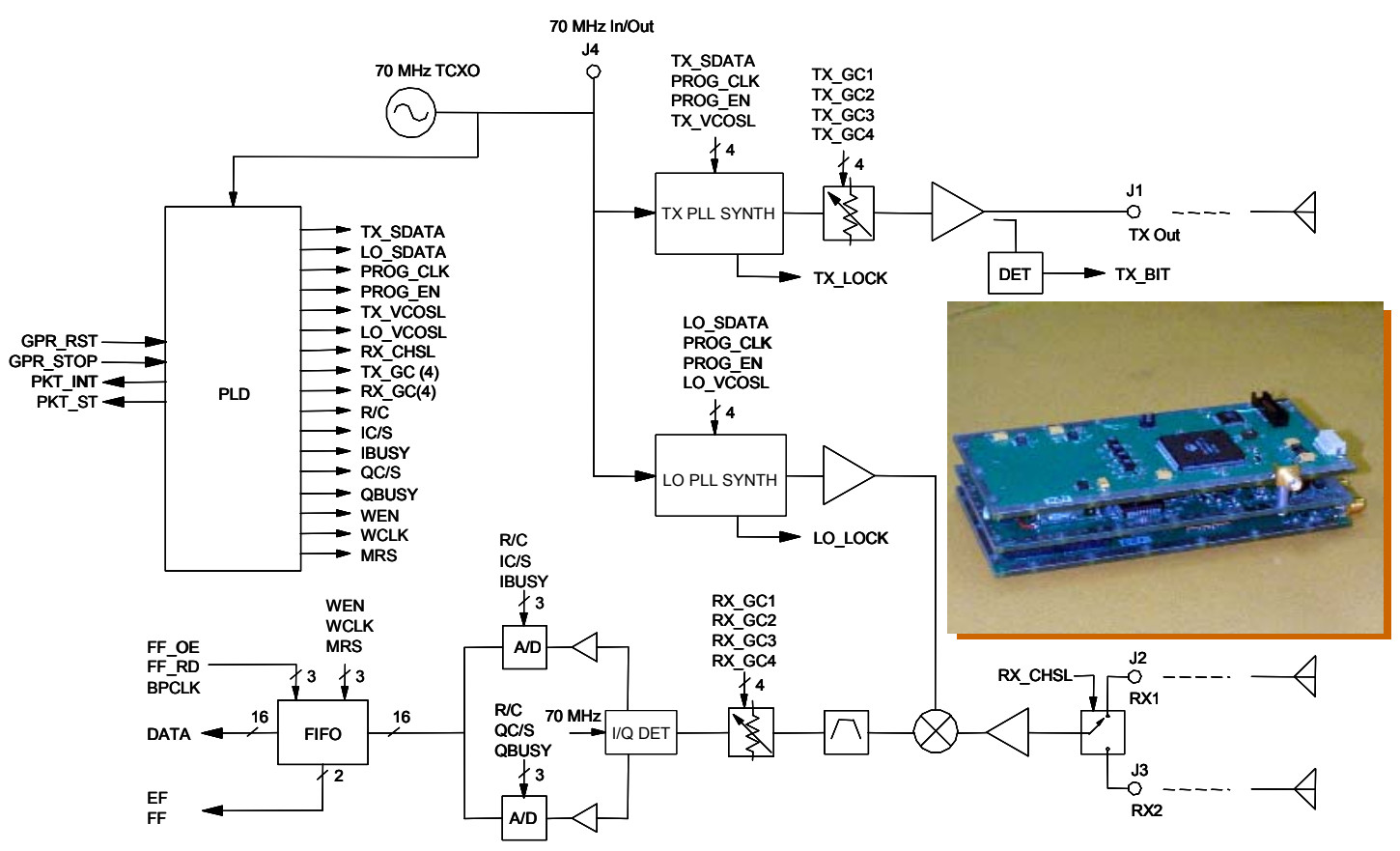

Figure 2.2-1. The CyTerra developed and tested RF module was used in the DOE effort with only minor component changes to meet the desired frequency range(s).

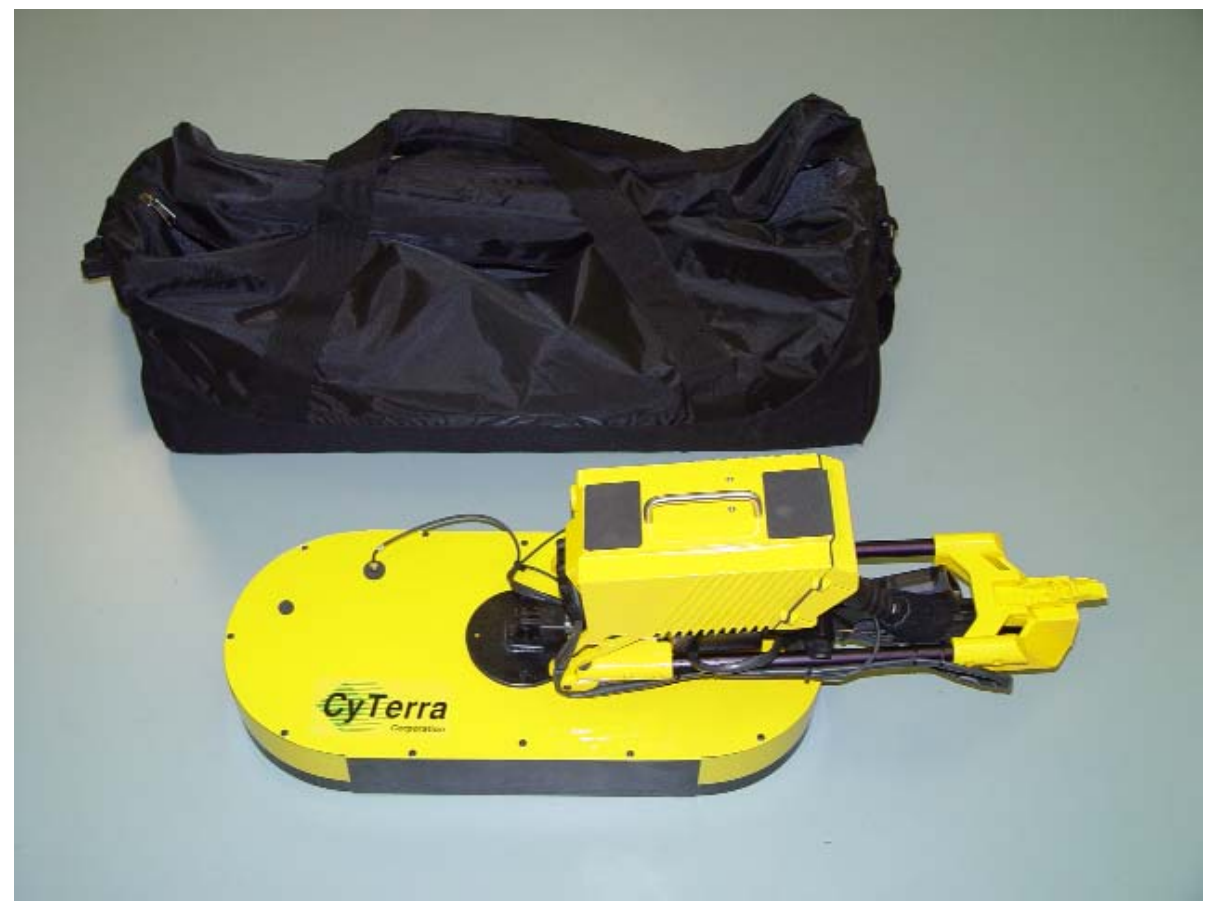

Figure 2.2-2. The compact, lightweight, foldout handheld detector is a key improvement. 


\subsection{Breakthroughs}

The engineering breakthroughs in this CyTerra effort resulted because of the hardware and performance breakthroughs already demonstrated for the US Army on buried plastic mine targets.

By exploiting the unique, comprehensive expertise CyTerra has gained through the US Army HSTAMIDS and related programs in detecting shallow buried non-metallic objects, CyTerra has provided for an engineering breakthrough in low cost, ease of use, and higher performance (better detection and lower false alarm rate). Only CyTerra has had the unique experience of accurately designing a low-cost, user friendly GPR system for difficult buried target detection through our comprehensive antenna and RF module design, as well as inventing and implementing sophisticated, beyond the state of the art buried target detection algorithms. Data collected at different depths for different pipe sizes and in different terrain, together with our adaptive/advanced algorithms, enabled prediction and (and eventually through follow-on testing), validation of performance for a range of potential operating frequencies. Customization and confirmation of projected capabilities using the handheld systems developed will enable proliferation of practical, low cost units as a breakthrough product.

\subsection{TECHNOLOGY DETAILS}

The scientific and technical basis and merit of the proposed work included the parametric data and modeling performed by CyTerra over the last 10 years that successfully led to Government field trials against buried plastic targets.

The technical basis for the DOE effort has been our knowledge of GPR design space for buried objects derived from our field test performance against buried plastic targets at last year's extensive US Army trials. We have developed, updated and correlated math models of GPR performance with soil and target characteristics, and have extended this model to the DOE depth and target types through use of the two GPR systems. Our models include cross-range as well as depth resolution, SNR, and can potentially be used to produce algorithm receiver operating characteristics (ROC) curves.

The legacy algorithm development from the US Army hand-held program was updated and extensively modified and enhanced for the prior LULU development effort. CyTerra demonstrated highly accurate plastic pipe detection down to three feet depth and only 3 inch diameter. (Additional parametric measurements included 1 inch and 2 inch plastic pies down to one foot and two feet, respectively.) The current algorithm includes cross polarization and copolarization data acquisition and processing. The amplitude and phase relationships for plastic and metal pipes was exploited to successfully eliminate clutter from buried rocks, roots, etc. The algorithm approach, shown in Figure 3.0-1, was demonstrated in real-time on the LULU system.

The current LULU algorithm massages the raw data to remove antenna side lobe effects by windowing in the frequency domain. (A Blackman filter is used on the cross pol data while a Chebyshev filter is used on the co pol data.). 


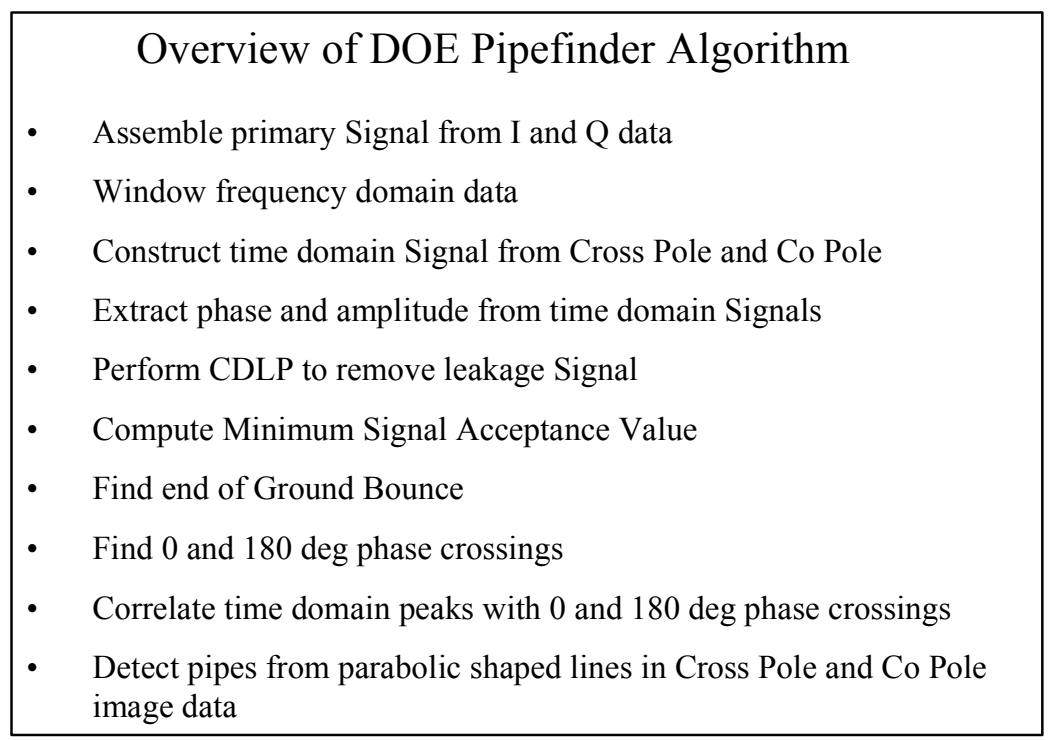

Figure 3.0-1. The Phase I algorithm demonstrated accurate small diameter (1 to 3 -inch) plastic pipe detection at depths between 1 and 3 feet.

The IFFT is then taken to obtain the cross pol and co pol time domain data (depth versus sample - Figure 3.0-2). The phase is unwrapped to remove discontinuities in the data, and then a linear prediction window filter is used across 100 samples to remove high DC levels and stretch the dynamic range to allow for the detection of the hyperbolas representing the radar returns from buried pipe (the CDLP - correlation detection linear prediction). This is done for both cross and co pol time domain images. Next, the ground bounce is removed and a minimum signal level (four sigma above the de level at each depth bin) is subtracted, leaving the input to the final algorithm steps. These final steps include independently inspecting the cross and co pol image data for consistent hyperbolic shaped lines that also follow 0 and 180 degree phase crossings and have amplitude peaks at the same locations. Finally, correlation between the cross and co pol results are used to indicate pipe detections (Figure 3.0-3).
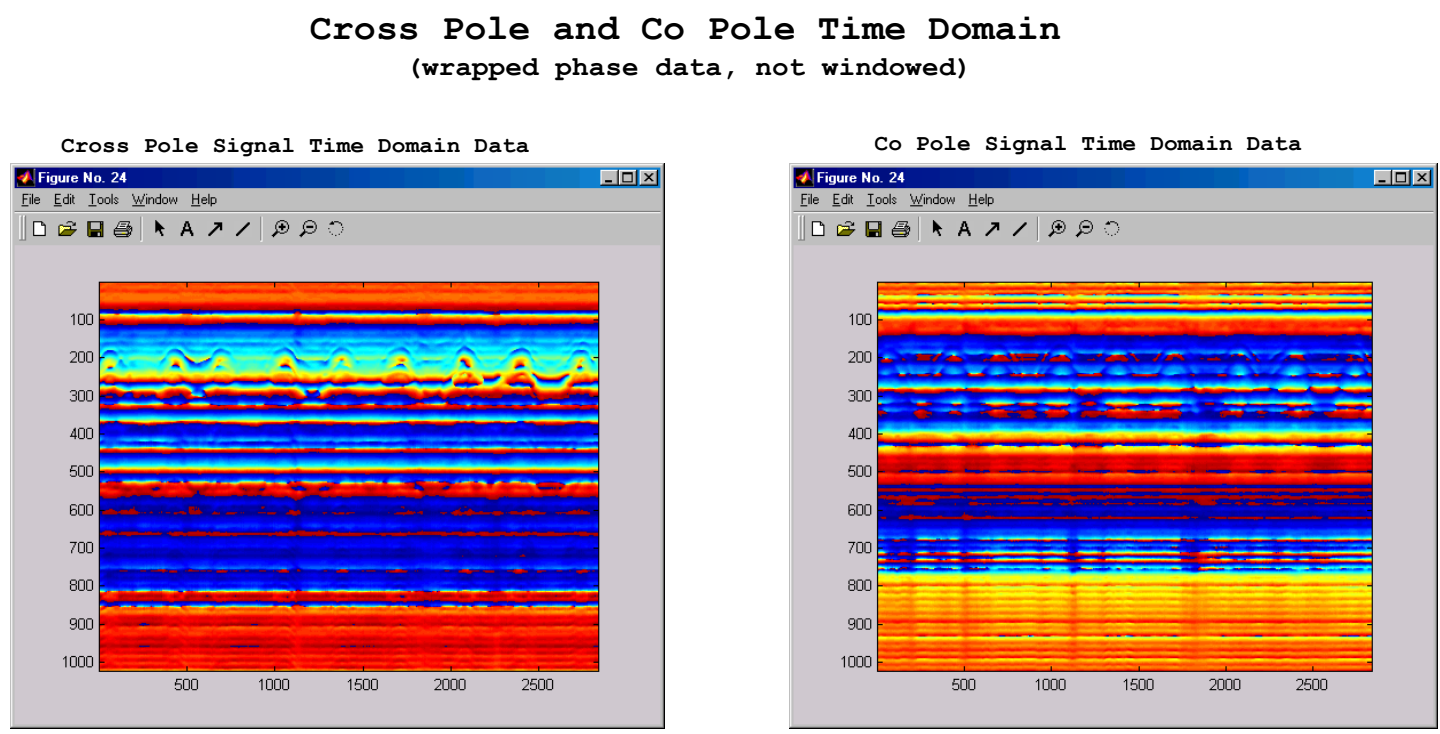

Figure 3.0-2. The Phase I algorithm initially data processing results in the time domain images for each polarization. 


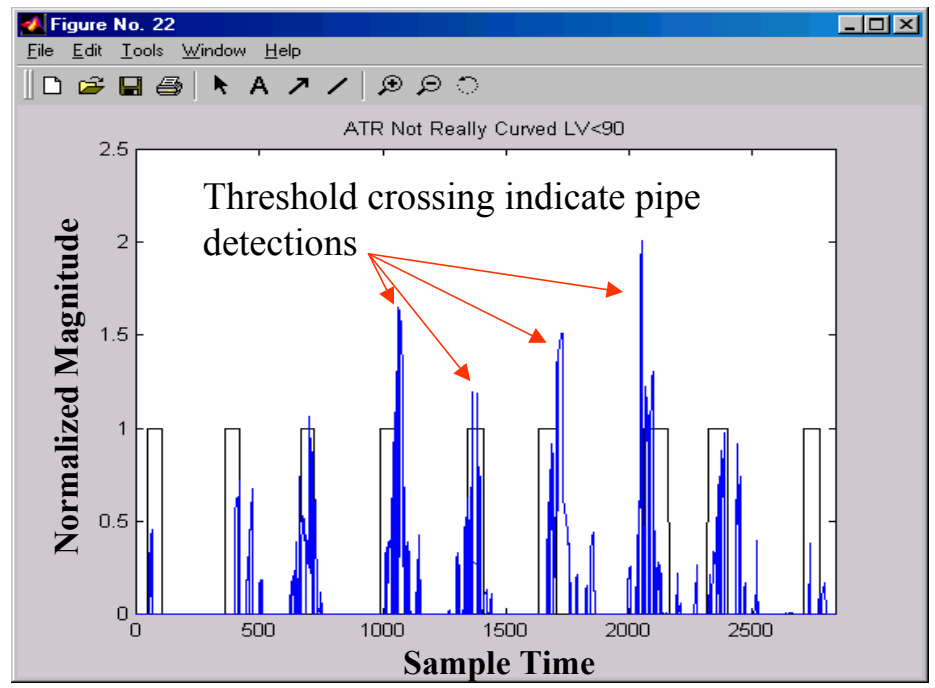

Figure 3.0-3. LULU detection of 6 inch plastic pipe at a depth of 2 feet

The key data that LULU provides includes broadband co-located dual-polarization amplitude and phase information. This information provides sufficient data for our algorithms to discriminate certain non-metallic objects (e.g., gas-filled plastic pipe) from metallic objects (e.g., metal pipe), and a flat plate from a cylindrical object. SAR processing can provide added crossrange resolution to determine the relative diameter of buried pipes. The next step is to apply signal-processing techniques that assist in the development of a "synthetic" image to convey pertinent target information to the user. This information will be modified from the actual radar imagery in order to provide only essential features necessary for an operator to make clear and concise decisions.

Detection of buried metallic or plastic objects pose a significant challenge. CyTerra understands the current state-of-art technology of GPR systems. Current short pulse or video GPR systems are sensitivity limited by problems with extraneous signal interference competing with the desired signal. The result is an unrecognizable signal, and even after processing and display, the data is unusable. The signal interference arises in most cases due to mismatches in components internal to the radar system, not from external sources. Some of the specifics are: (1) antenna mismatch due to bandwidth limitations and dispersion; (2) transmitter waveform sidelobes; (3) multiple transmitter/receiver/antenna reflections due to mismatch impedances; and (4) non-uniform bandwidth, no absolute control of transmitter. The CyTerra frequency stepped GPR system design that eliminates the problems of the current 'short-pulse' radar systems allows for an increase in measurement capability. Bandwidth control improves resolution needed for the location of small non-metallic objects/small cross-section plastic objects and a reduction in interference improves sensitivity and signal clarity.

The CyTerra frequency-stepped radar permits operation at an RF duty factor approaching unity, to remove the short-pulse radar requirement that the RF equipment (transmitter, antenna, and receiver) be instantaneously broad band. It also achieves a fully coherent radar capability while retaining (and expanding) the achievable high-range resolution capability. The significance 
of the high RF duty factor is that the thermal noise limited detection sensitivity of the radar can be achieved using readily available components, as demonstrated in the HSTAMIDS program. In fact, with the high RF duty factor stepped frequency waveform. The sensitivity limit is dictated by parameters related to the soil/earth environment in which objects are located.

The frequency-stepped radar is fully coherent which allows for compensation of hardware amplitude and phase (dispersion) errors over the operating RF band, and permits two- and threedimensional synthetic aperture image processing to be accomplished.

\subsection{GPR Hardware}

A system level block diagram for the frequency-stepped GPR electronics is given in Figure 3.1-1. Configured is a bi-static system (separate transmit and receive antennas) with appropriate calibration and signal processing. The critical technology advantage for the GPR system proposed lies in the bandwidth control, resultant sensitivity increase and the antenna match that can be achieved given the frequency bandwidth that must be covered and the various soil conditions through which the antenna must propagate energy. Figure 3.1-2 gives the overall radar specifications.

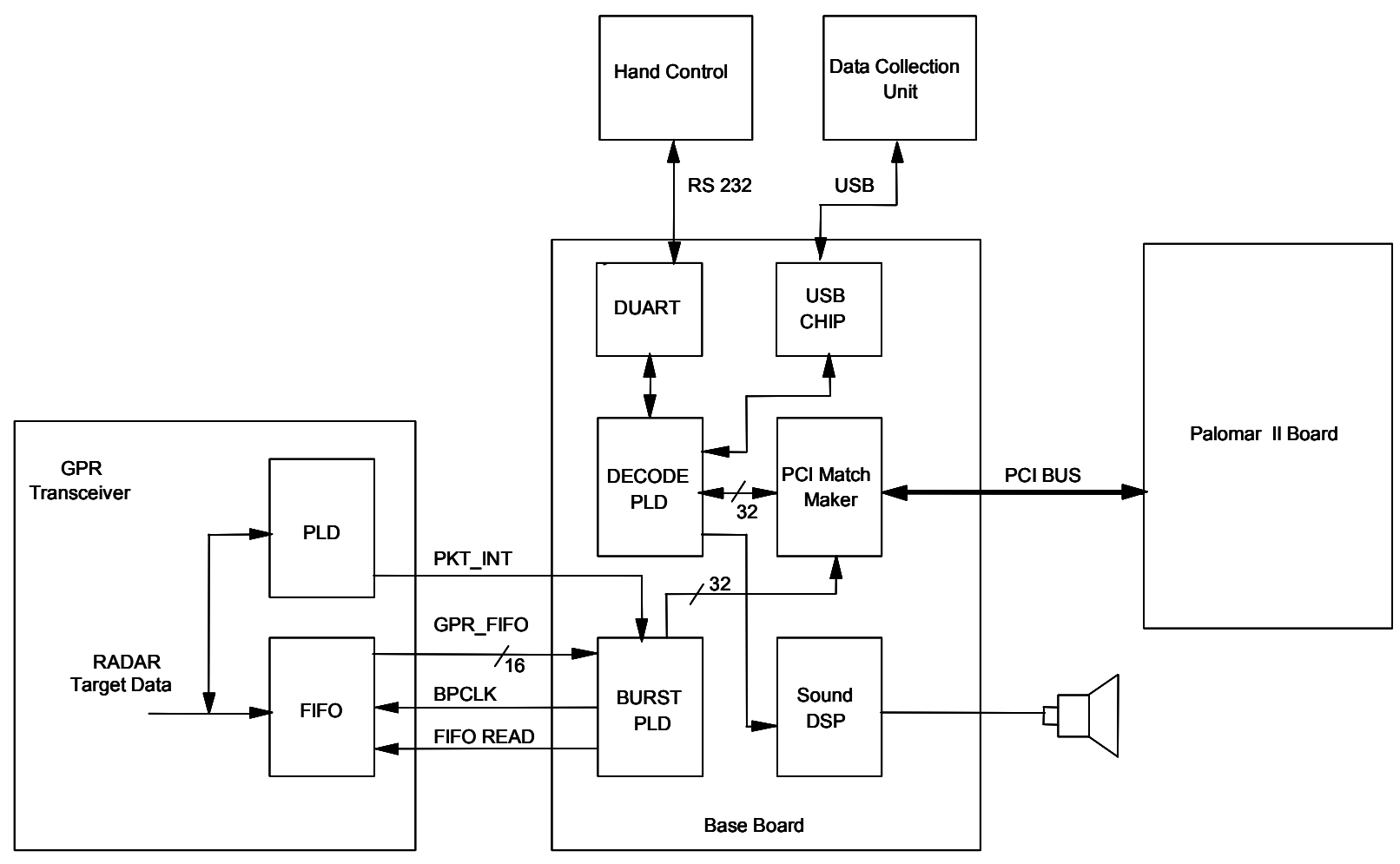

Figure 3.1-1. System electronics block diagram for the LULU system is identical to the field proven HSTAMIDS. 
- Radar type

- Antennas

- Frequency band

- Step size

- Switching speed

- Measurement Rate

- Power output transmit

- Power

- Battery Life

- Weight
Frequency stepped

12-inch / 9-inch spirals

200-800 MHz / 400-1500 MHz

$10 \mathrm{MHz}$

100 microseconds / frequency

$90 \mathrm{HZ}$

30 milliwatts, maximum

12 Vdc, 3 amps

4 hours, single charge

$11 \mathrm{lbs}$

Figure 3.1-2. LULU system parameters cover the frequencies of interest in lightweight, low-cost, handheld system(s).

\subsection{Antenna Design}

From the viewpoint of size reduction, the spiral antenna is the most attractive. For an antenna to be an efficient radiator, it must normally have a dimension of at least one-half wavelength. The spiral radiates efficiently when it has an outer circumference of at least one wavelength. This means it needs a maximum diameter of about one-third wavelength. The upper frequency limit for efficient spiral radiation is set by the size of the feed point attachments, and the lower frequency limit is set by the outer diameter of the spiral structure. Within these limits, the spiral radiates efficiently in a frequency-independent manner. The input impedance and the radiation patterns will vary little over this frequency range. With any spiral structure, an upper frequency of several gigahertz presents no problem.

The spiral antenna is constructed by etching the pattern on a printed circuit board. A planar, printed circuit, spiral antenna radiates perpendicular to the plane of the spiral. The spiral itself is located at the end of a cylindrical metal cavity (the cavity back) to provide isolation from neighboring elements and electronics. Typically, an absorber is used on the top-side of the spiral inside the cavity to make sure the element responds only downward. Separate transmit and receive antennas are used to simplify the electronics, provide spatial separation and reduce very shallow reflections.

\subsection{Digital Hardware and Software}

To meet the HSTAMIDS processing requirements the PowerPC 750 processor on a PMC mezzanine form factor was used. Processing algorithms for HSTAMIDS utilized less than 50\% of the processing capability of the PowerPC 750 running at $350 \mathrm{MHz}$. Since the data rate of the DOE sensor suite is about the same as that of HSTAMIDS, the same hardware was used.

Using this existing hardware, the throughput requirements for HSTAMIDS and the management of the computationally intensive tasks of initial construction and subsequent 
maintenance of the Principal Component clutter model was accomplished. The throughput for the DOE LULU pipefinder was overall about the same as that of HSTAMIDS. In addition to the audio output, the DOE system could later (in follow-on efforts) include digital signal processing algorithms to process the sensor information to obtain a composite object shape.

\subsection{TASKS AND RESULTS}

The CyTerra team handheld detector is a very capable buried pipe alert system. Our radar technology provides real-time alerts, with the potential for high resolution 2- and 3-dimension maps through the use of very wide bandwidth and coherent synthetic aperture imaging algorithms.

Using HSTAMIDS equipment (the GPR and the portable data recorder), CyTerra collected buried plastic pipe signatures in various soils and at various depths. Specific locations, pipe diameters, and pipe depths were selected after initial discussions with the DOE customer on his desires/needs. After designing and building a customized handheld GPR meeting DOE needs, we collected buried plastic pipe signatures in various soils and at various depths.

The hardware was developed from selection of off-the-shelf VCOs that meet the low and high frequency bands (200 to $800 \mathrm{MHz}$ and 650 to $1600 \mathrm{MHz}$ ) and included redesign and relayout of the proven HSTAMIDS RF module in two designs, to meet the DOE frequency ranges. Two derivative RF modules were fabricated at the two DOE frequency ranges, including spare boards and housings. The HSTAMIDS design was be used, including the housing, to help meet schedule and cost goals. Housings, antennas, electronics, control software and algorithm (target detection) software were integrated and lab tested against sandbox targets for functionality (Figure 4.0-1 and 2).

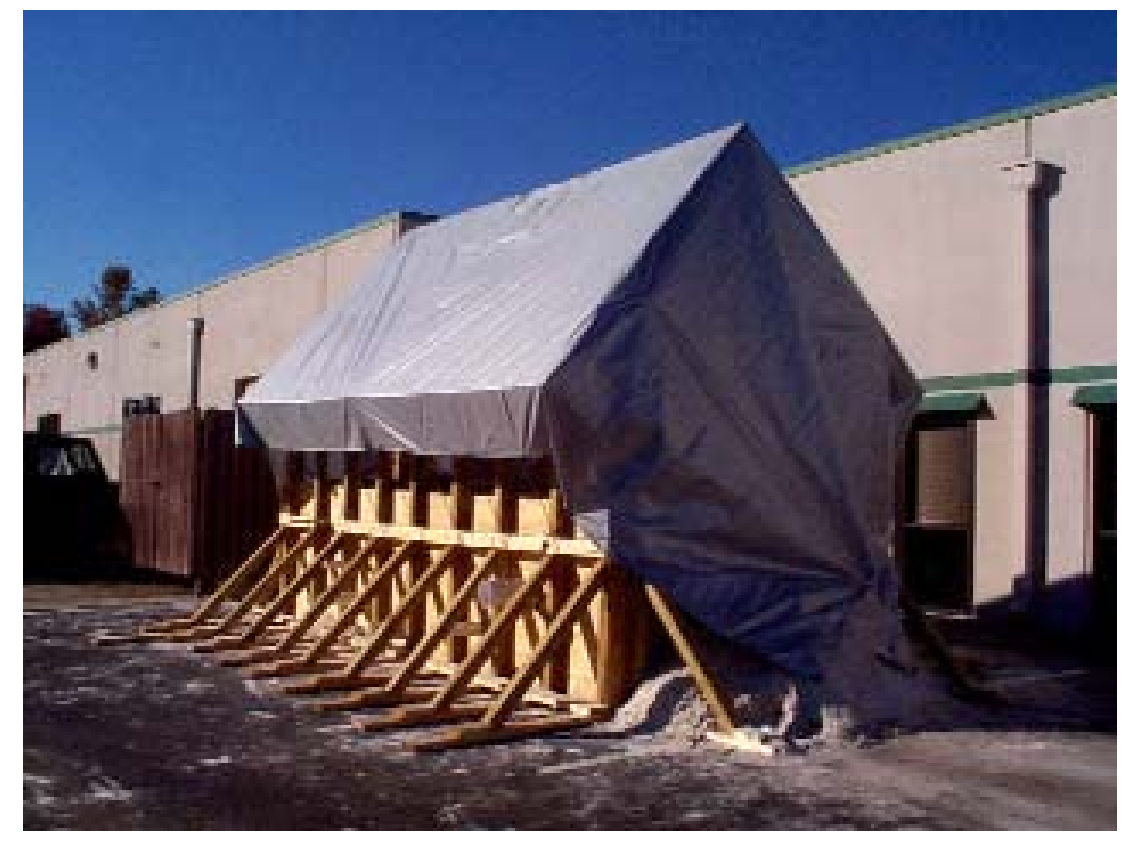

Figure 4.0-1. The sandbox was interlaced with several plastic pipe targets to allow for early hardware and algorithm tests prior to field testing 


\section{“As Built” Measured Pipe Locations}

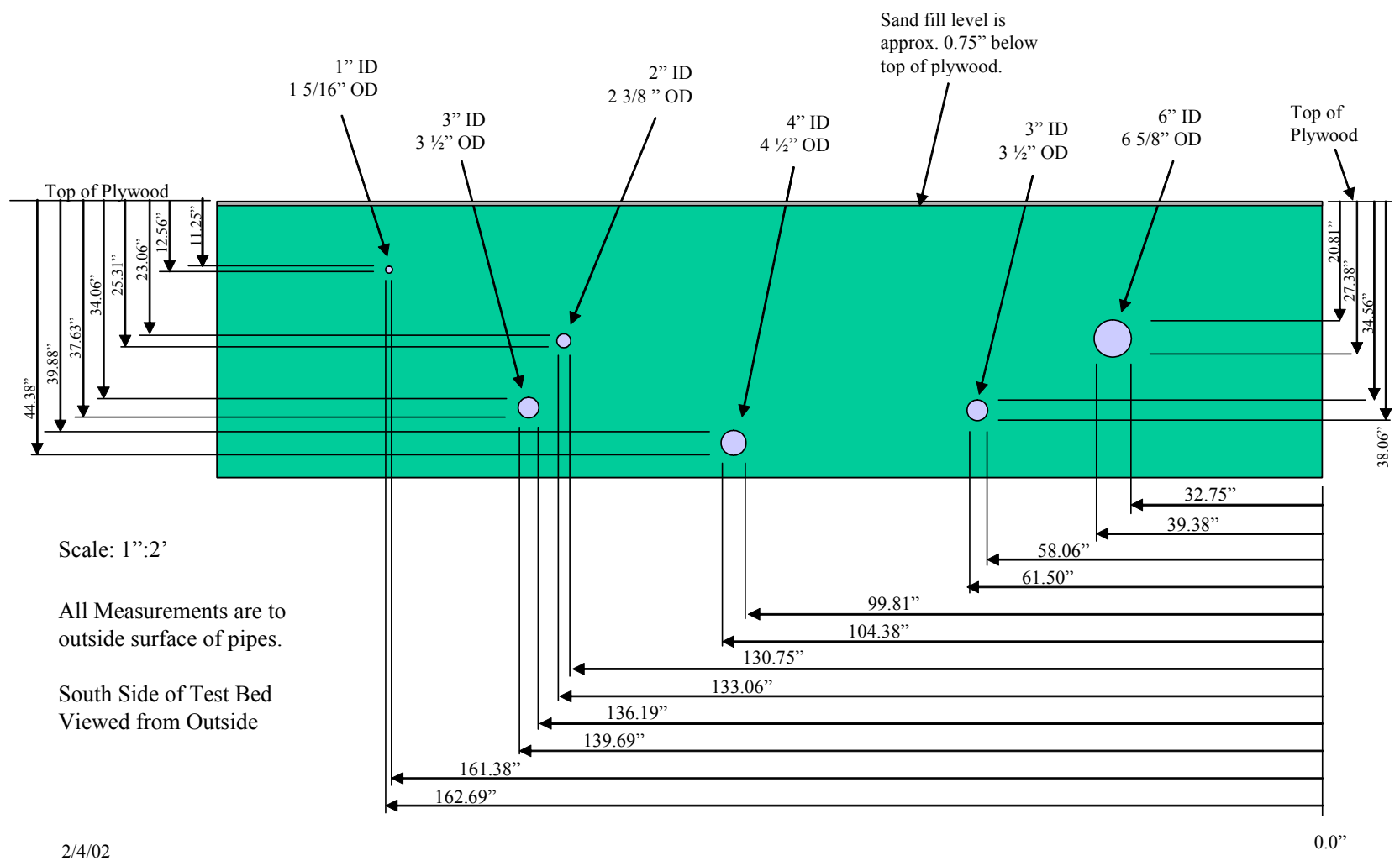

Figure 4.0-2. Pipe locations in the sandbox

Two sets of antennas were sized, drawn and fabricated; one for the low frequency system and the second for the high frequency system. Except for size, they are copies of the proven spiral antenna design from HSTAMIDS.

The LULU algorithms were developed from redefined HSTAMIDS algorithms to include the DOE selected targets at selected depths and soils. Effects of the different frequencies were also accounted for, with initial sandbox measurements promising (Figure 4.0-3). CyTerra updated the code to meet the new algorithm definitions including an attempt at balancing the Pd versus FAR according to the anticipated pipe finding needs. Closed loop testing on stored data using desktop computers communicating via Ethernet was also conducted to ensure proper validation when transitioning to the portable, hand held hardware.

CyTerra conducted tests against a different set of targets in our backyard (Figures 4.0-4 and 5) to validate or find necessary fixes to the lab and sandbox tested code and hardware show the layout of buried pipes in our backyard. 

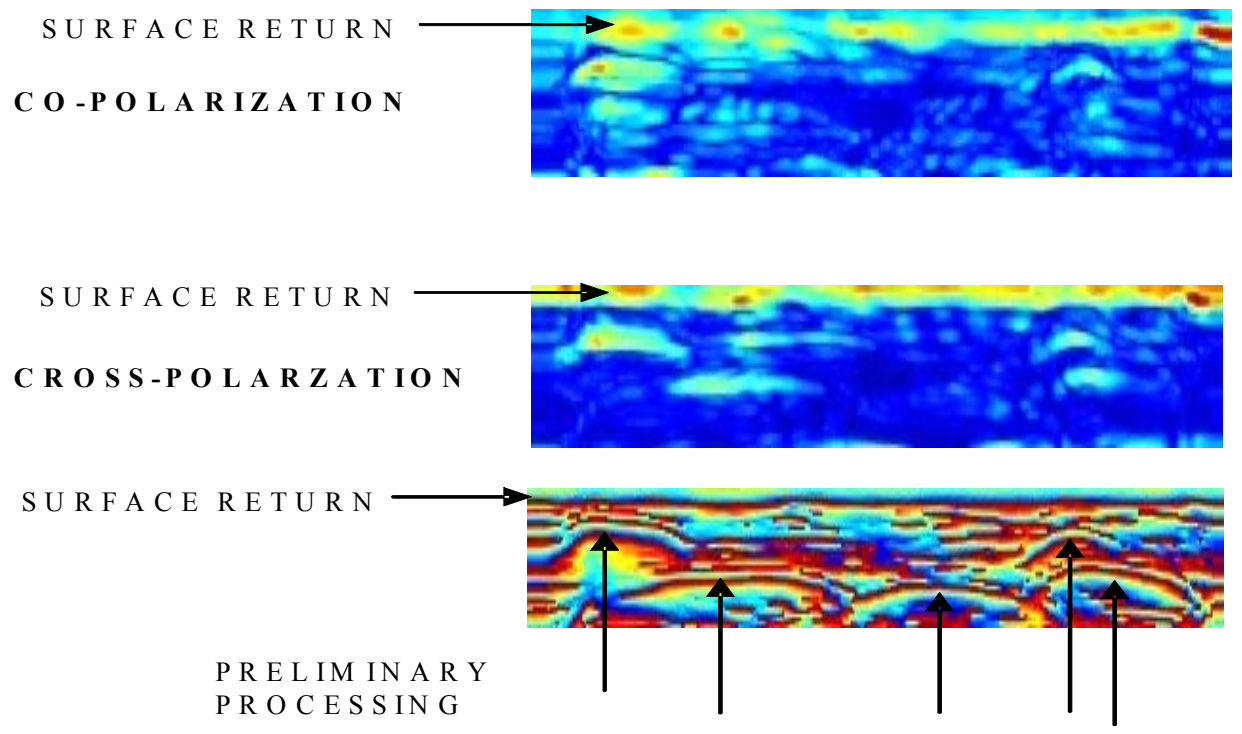

PLASTIC PIPE L O C A TION S

Figure 4.0-3. Sandbox measurements indicated good capability for detection of buried plastic pipes
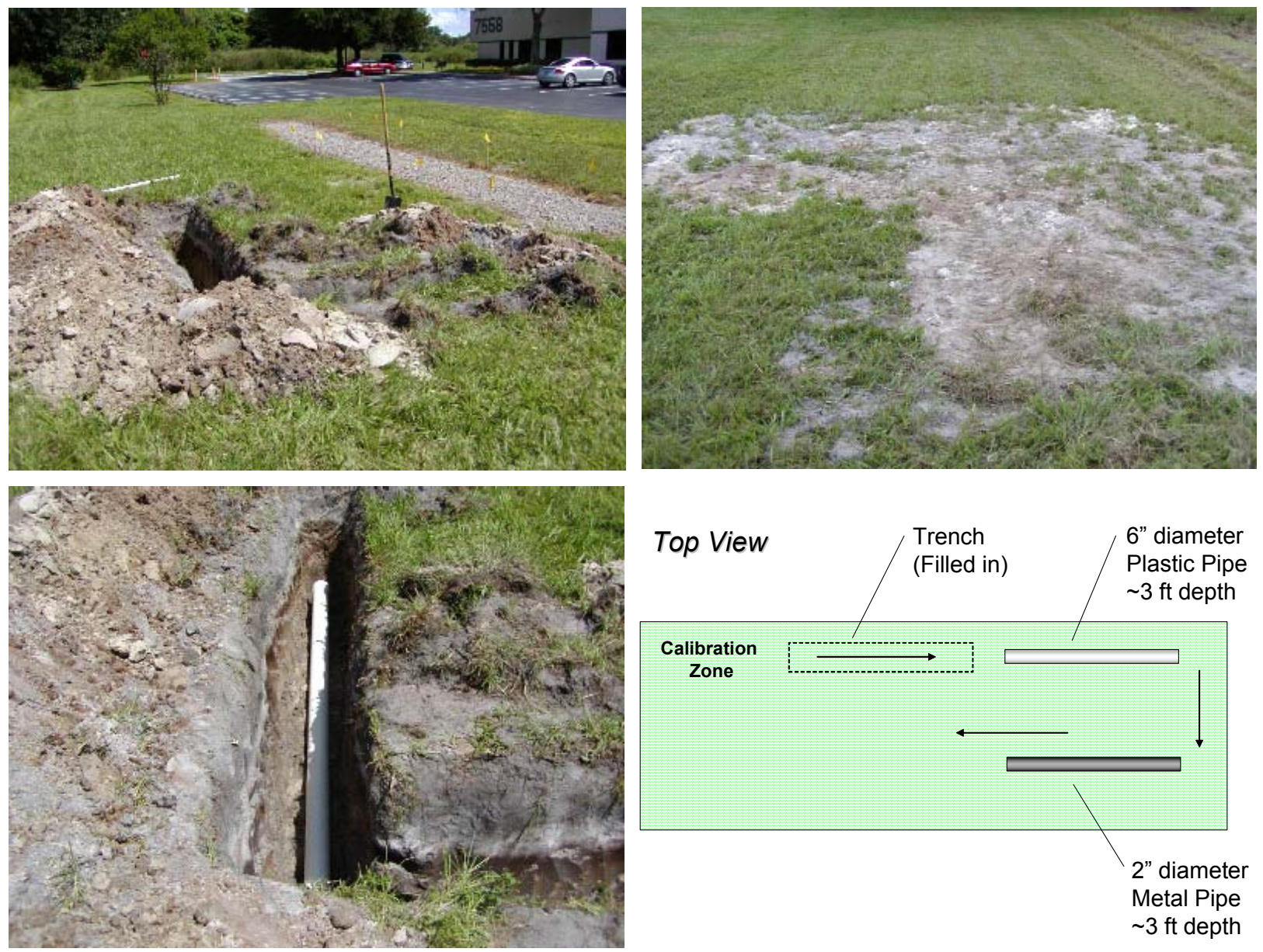

Figure 4.0-4. Backyard trench and pipes were buried for post-sandbox data collection and test 

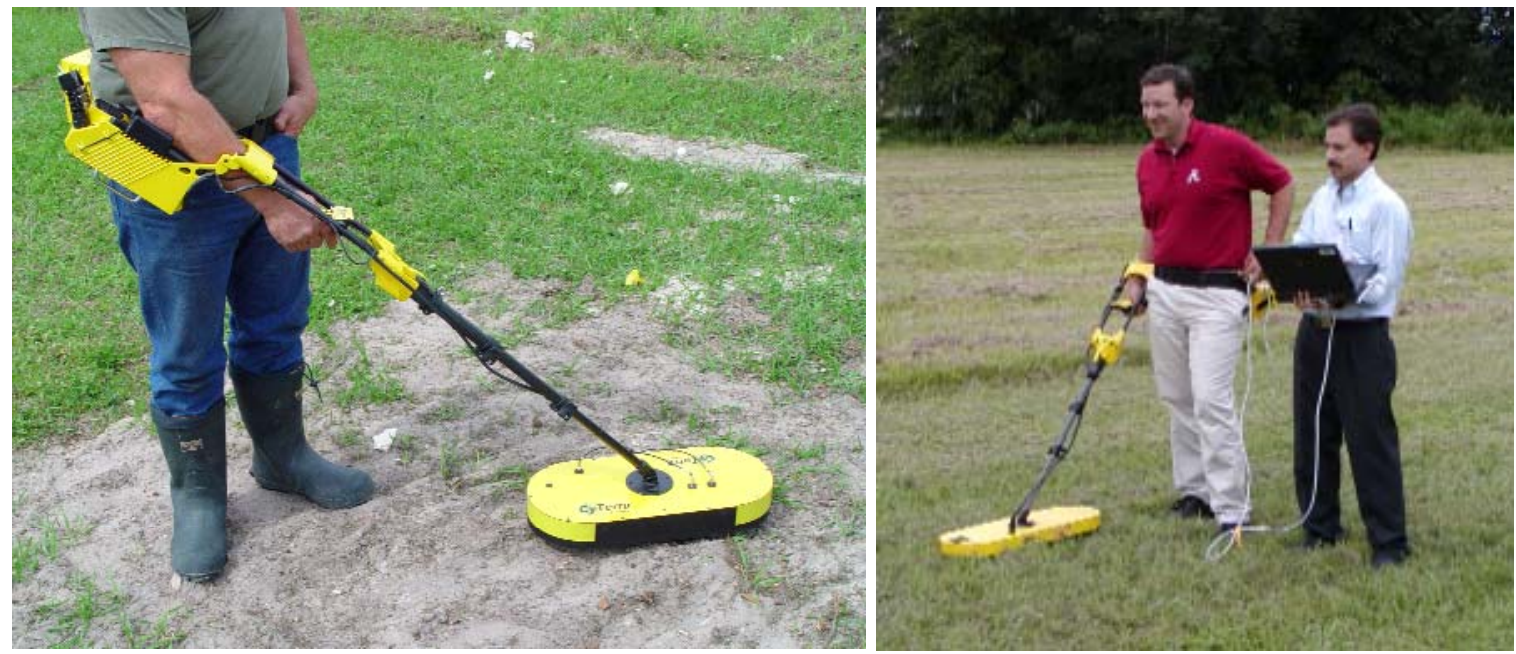

Figure 4.0-5. Early and later measurements and tests addressed freshly buried verses weathered in targets.

A 2D offline image is available to indicate the depth of the detected pipe (Figure 4.0-6). This is a user-friendly implementation; unlike existing depth plots that require operator interpretation to both detect and range the pipe location, the CyTerra system will have already alerted the operator to the location through audio.

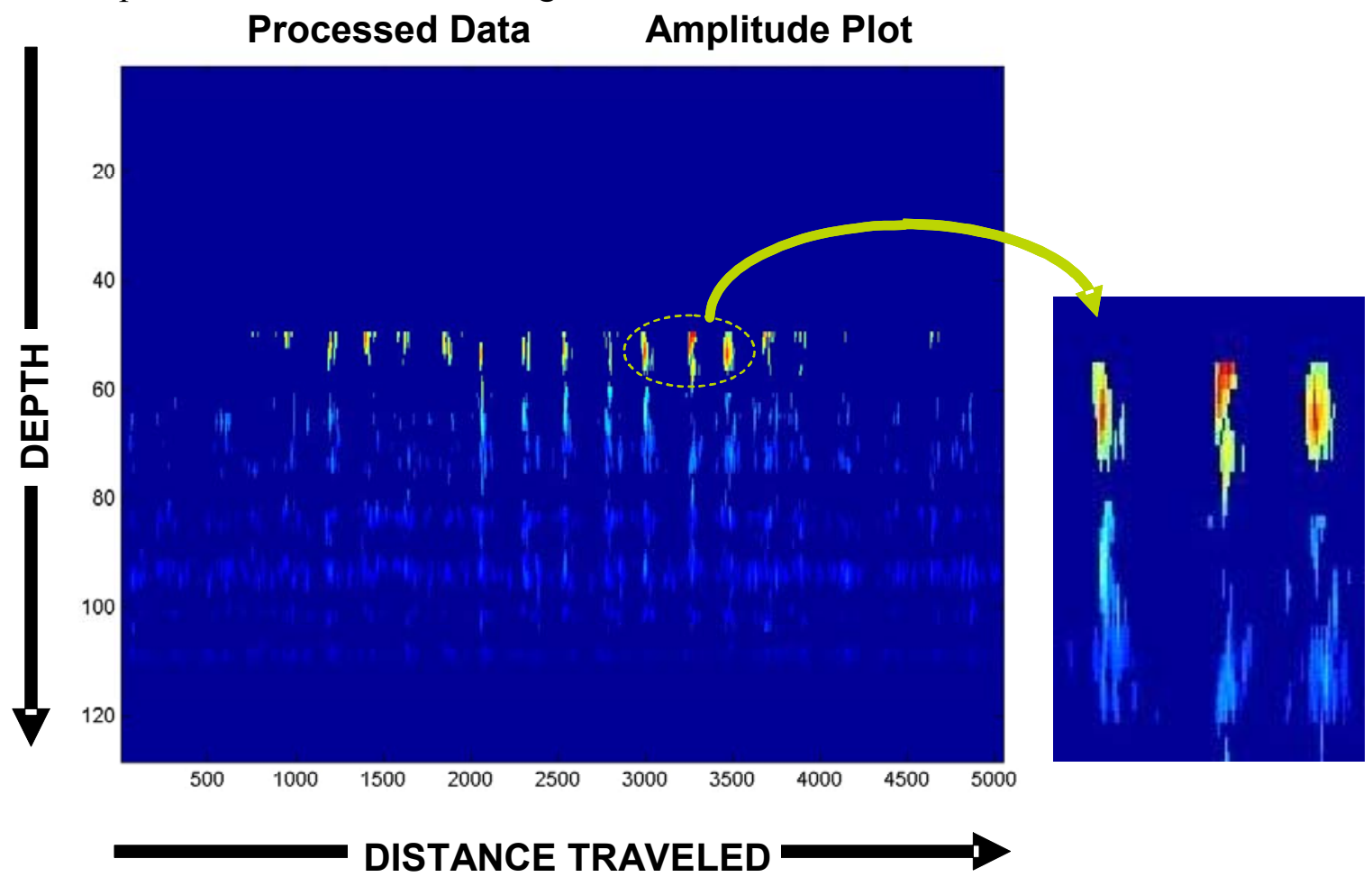

Figure 4.0-6. Amplitude plot shows pipe locations as the operator repeatedly swings over it while advancing 
Real-time audio detection is available because our multi-threaded processing permitted the necessary simultaneous operation of the HSTAMIDS advanced ATR algorithms and the GPR control code, system tasks, and other tasks in the background. The current HSTAMIDS processing of GPR data relies on Principal Component Analysis (PCA) performed automatically upon appropriately selected and conditioned features of the GPR response in clutter and mine environments (Figures 4.0-7 through 4.0-9). For the DOE problem, CyTerra has seen significantly better performance because of the extent (length) of the pipe targets versus the smaller mine targets.
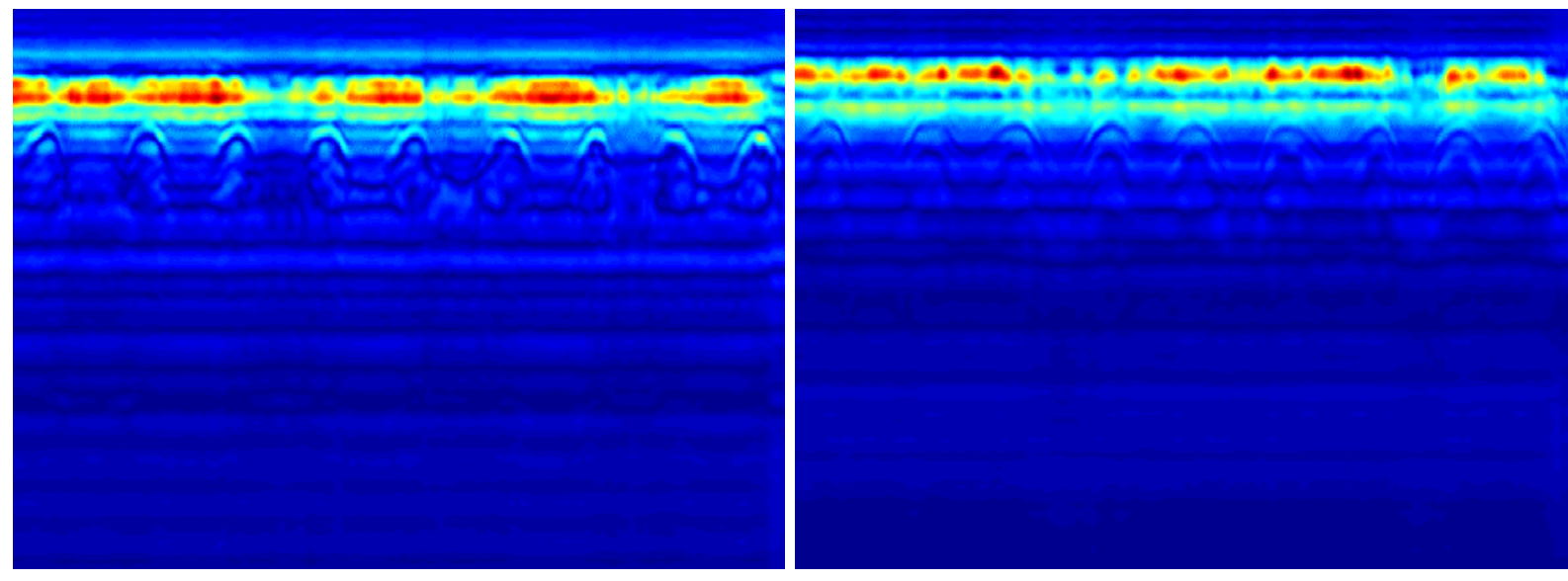

Figure 4.0-7. Backyard cross-pol (left) and co-pol depth plots show the customary hyperbolic locations of the 4 inch plastic pipe buried at 4 feet.

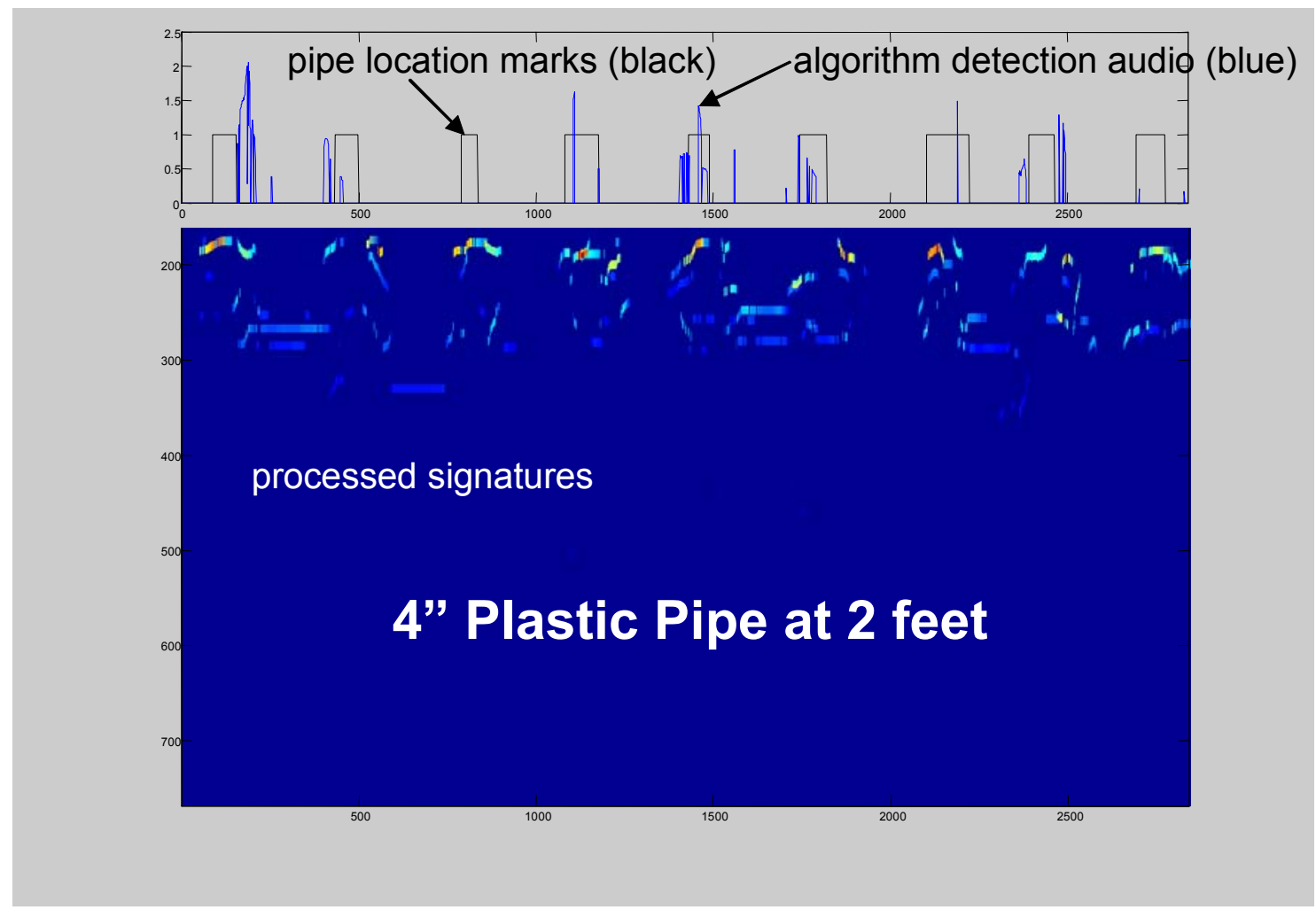

Figure 4.0-8. Full algorithm audio alerts show accurate pipe locations 


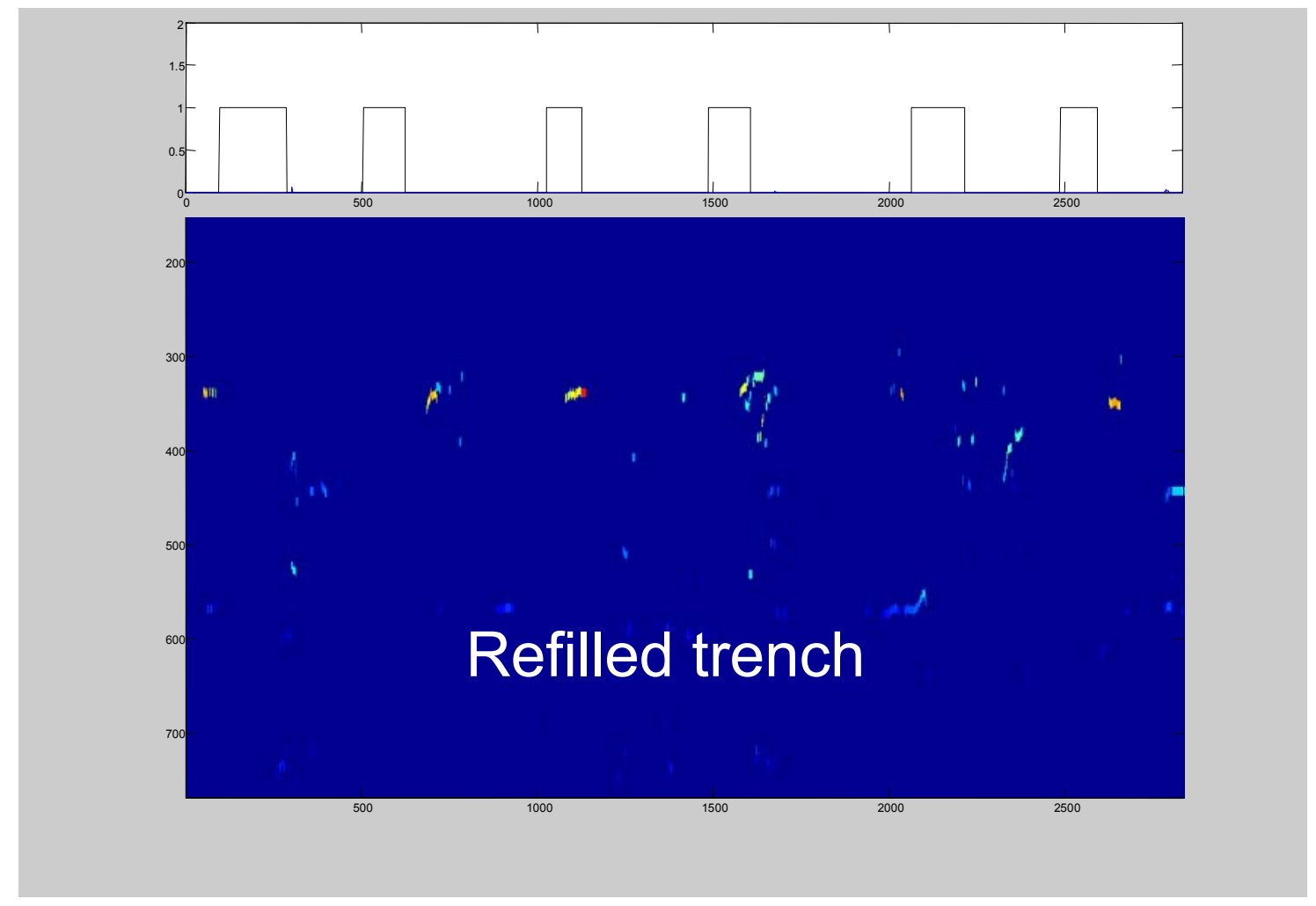

Figure 4.0-9. LULU operation over refilled trench shows no false alarms

CyTerra also prepared detailed briefings for presentation to the Contracting Officer's Representative (COR). In addition, CyTerra provided and presented a technical paper at the DOE/NETL Annual Contractor's Review Meeting.

\subsection{CONCLUSIONS}

As shown in Figure 5.0-1, CyTerra has been largely successful in making a user friendly LULU system. Follow-on efforts to determine the breadth of its accuracy in other soils, and addition of visual displays to complement the audio, remain to be performed.

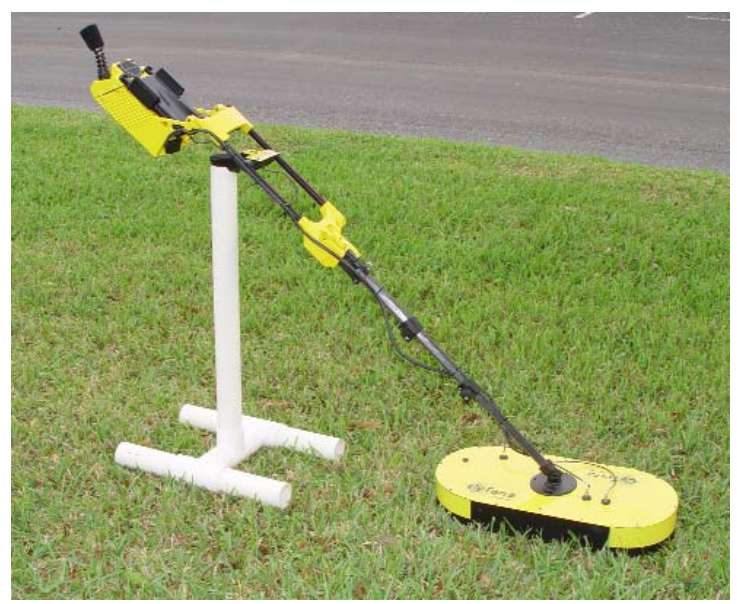

- Side-to-side scans in area of interest

- Operator travels along path of detections

- Operator follows pipe and marks line of detection

- Method of operation is similar to existing location devices

- Low level of decision making required from operator

- System is handheld and stores quickly and easily

Figure 5.0-1. Successful user friendly buried plastic pipe detection has been demonstrated by CyTerra in local sandbox and backyard locations using $L U L U$ 